Check for updates

Cite this: Soft Matter, 2017, 13, 4294

Received 22nd March 2017 Accepted 15th May 2017

DOI: 10.1039/c7sm00572e

rsc.li/soft-matter-journal

\title{
Lipid rafts enhance the binding constant of membrane-anchored receptors and ligands
}

\author{
Long $\mathrm{Li}^{,}{ }^{\mathrm{a}}$ Jinglei Hu, (D) *bc Xinghua Shi, (D) de Yingfeng Shao ${ }^{\mathrm{a}}$ and Fan Song (D) *ae
}

\begin{abstract}
Gaining insights into the binding of membrane-anchored receptors and ligands that mediate cell adhesion and signal transduction is of great significance for understanding numerous physiological processes driven by intercellular communication. Lipid rafts, microdomains in cell membranes enriched in cholesterol and saturated lipids such as sphingomyelin, are believed to serve as the essential platforms to recruit protein molecules for biological functions. An important question remains how the lipid rafts affect the binding constant of membrane-anchored receptors and ligands. We have investigated the adhesion of multicomponent membranes by using Monte Carlo simulations of a mesoscopic model with biologically relevant parameters. We find that the preferential partitioning of membrane-anchored receptor and ligand proteins in the lipid rafts significantly increases the binding constant of those proteins, in cooperation with the shape fluctuations of the membranes caused by thermal excitations. The binding constant can even be greater than that of the same receptors and ligands anchored to two apposing supported, planar membranes without shape fluctuations. The membrane shape fluctuations facilitate the binding of the anchored receptors and ligands, in contrast to the case of homogeneous membranes. Our results suggest that cells might regulate the binding of membrane-anchored receptor and ligand proteins by modulating the properties of lipid rafts such as area fraction, size and the affinity of rafts to the proteins.
\end{abstract}

\section{Introduction}

Cell adhesion and signal transduction regulate many important biological processes such as immune response and tissue development via the specific binding of receptor and ligand molecules anchored to the two apposing cell membranes. ${ }^{1,2}$ The key property to quantifying the adhesion behaviors and adaptive immune responses ${ }^{3,4}$ is the binding equilibrium constant of these membrane-anchored protein molecules

$$
K=\frac{[\mathrm{RL}]}{[\mathrm{R}][\mathrm{L}]},
$$

where $[\mathrm{R}],[\mathrm{L}]$ and $[\mathrm{RL}]$ are the area concentrations of unbound receptors, unbound ligands and bound receptor-ligand complexes, respectively. Given the importance of the two-dimensional binding constant, a variety of experimental techniques have been utilized to

\footnotetext{
${ }^{a}$ State Key Laboratory of Nonlinear Mechanics (LNM) and Beijing Key Laboratory of Engineered Construction and Mechanobiology, Institute of Mechanics, Chinese Academy of Sciences, Beijing, 100190, China. E-mail: songf@lnm.imech.ac.cn

${ }^{b}$ Kuang Yaming Honors School, Nanjing University, Nanjing, 210023, China. E-mail: hujinglei@nju.edu.cn

${ }^{c}$ Shenzhen Institute of Research, Nanjing University, Shenzhen, 518057, China

${ }^{d}$ National Center for Nanoscience and Technology, Beijing, 100190, China

${ }^{e}$ School of Engineering Science, University of Chinese Academy of Sciences, Beijing, 100049, China
}

characterize the binding constant, such as fluorescence spectroscopy, ${ }^{5-8}$ micro-pipette aspiration assay, ${ }^{9-11}$ atomic force microscopy, ${ }^{12-14}$ and flow chamber. ${ }^{15-17}$ Due to different physiological conditions in those experiments, the as-measured binding constant of the same receptors and ligands can differ by orders of magnitude. ${ }^{18,19}$ Recent molecular dynamics simulations have revealed that the binding constant depends strongly on the nanoscale roughness of the membranes caused by thermally-excited shape fluctuations. ${ }^{20-24}$

In the aforementioned studies, the biomembrane is often viewed as a fluid bilayer with a homogeneous distribution of lipids and proteins as proposed by the Singer-Nicolson model 40 years ago. ${ }^{25}$ The paradigm of biological membranes has gone through a major update. Cumulative evidence indicates that the membranes are not structurally homogeneous, but rather consist of microdomains enriched in saturated phospholipids and cholesterol. These microdomains, termed as lipid rafts, exist as distinct liquid-ordered phases that float freely as stable entities in the liquid-disordered matrix of the plasma membrane. ${ }^{26-29}$ Lipid rafts can lead to a heterogeneous distribution of proteins in the membranes by recruiting them to variable extents, see Fig. 1(a). In addition, lipid rafts often exhibit less fluidity than the surrounding membranes, due to the compact packing of the hydrophobic chains of saturated lipids. ${ }^{31,32}$ It is generally believed that lipid rafts serving as 


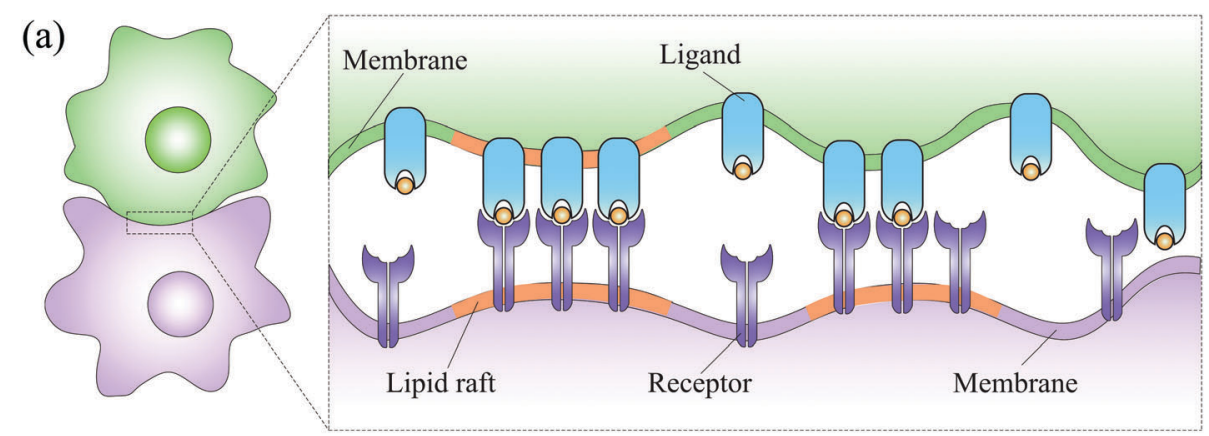

(b)

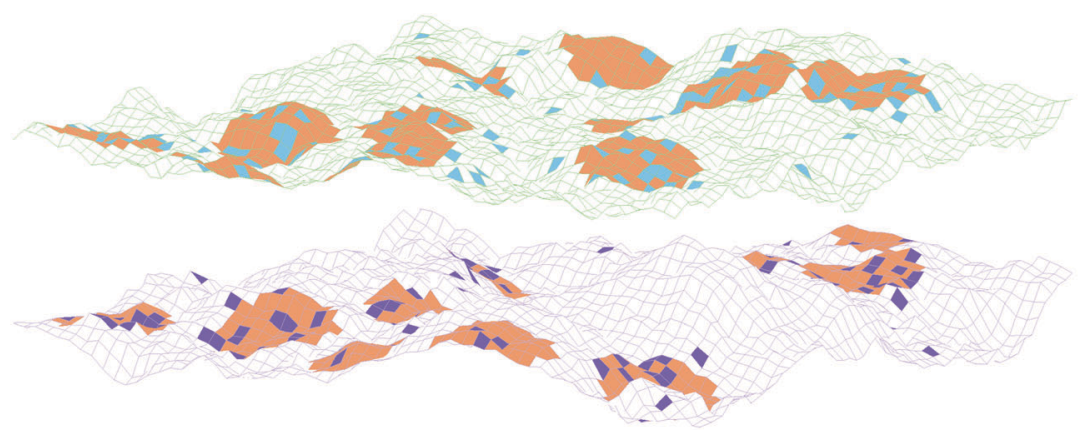

Fig. 1 (a) Cartoon of the contact zone of two adhering cells. The lipid raft regions of the cell membranes are depicted in orange. (b) Snapshot of two fluctuating model biomembranes interacting via receptor-ligand binding obtained from our Monte Carlo simulations. The lipid raft domains are represented by the clusters of orange patches. The receptor and ligand proteins are described by the light purple and blue sites, respectively. Both the raft domains and proteins diffuse along the membrane surfaces. One protein molecule occupies a single membrane patch. A receptor binds to a ligand only when they are apposing to each other on the two membranes and within the binding range (see eqn (3)). Anchored proteins often generate curvature on the membranes. ${ }^{30}$ In this model, the receptors and ligands do not induce spontaneous curvature. The humps of the membranes arise from the thermal excitations. The clustering or aggregation of the proteins on the lipid rafts is reflected by the affinity $\Delta U$ of the raft to the proteins as described in the Model and method section.

signaling platforms can facilitate protein-protein interactions on a single membrane by virtue of spatial proximity of participating components. ${ }^{26,27,31,33}$ Only a few in situ experimental studies ${ }^{4,34,35}$ have reported that the lipid rafts help the binding of $\mathrm{T}$ cell receptors (TCR) to peptide-major histocompatibility complexes (pMHC) anchored to antigen-presenting cell membranes. The role of lipid rafts in the binding of membrane-anchored receptors and ligands yet remains to be quantified.

In this paper, we report Monte Carlo (MC) simulations of multicomponent membrane adhesion. We systematically varied the area fraction and size of the lipid rafts on the membranes, the affinity of the rafts to the anchored receptors and ligands, and the bending rigidity of the raft domains of the membranes. The binding constant of the membrane-anchored receptor and ligand protein molecules is found to be enhanced in the presence of lipid rafts. This enhancement can be attributed to the entropy gain of the membranes resulting from raft-induced protein aggregation. The thermal shape fluctuations of the membranes actually make a positive contribution to the binding constant of the anchored proteins. For the lipid rafts with a size of around $100 \mathrm{~nm}$ and an area fraction of $10-15 \%$, typical values in experiments, the binding constant is surprisingly even greater than that of the same receptors and ligands anchored to two apposing supported, planar membranes without shape fluctuations. In addition, we find that the increase of bending rigidity of the lipid rafts helps the aggregation of proteins and therefore facilitates the binding. Our results suggest that cells might regulate the binding of membrane-anchored receptor and ligand proteins by modulating the properties of lipid rafts such as area fraction, size and the affinity of rafts to proteins.

\section{Model and method}

We start with the classic mesoscopic model of membrane adhesion. ${ }^{20,36,37}$ The two membranes are represented by twodimensional square lattices with the Helfrich elastic energy given by

$$
\mathcal{H}_{\mathrm{be}}=\sum_{i} \frac{\kappa_{i}}{2 a^{2}}\left(\Delta_{\mathrm{d}} l_{i}\right)^{2}
$$

where the lattice size $a$ sets the length unit, $\kappa_{i}=\kappa_{1, i} \kappa_{2, i} /\left(\kappa_{1, i}+\right.$ $\left.\kappa_{2, i}\right)$ is the effective bending rigidity of the two apposing patches at lattice site $i$, and $\Delta_{\mathrm{d}} l_{\mathrm{i}}$ is the discrete Laplacian of the local membrane separation $l_{i}$. One receptor or ligand occupies a single square patch in this model. The adhesion energy from the binding of receptors and ligands on the two membranes assumes

$$
\mathcal{H}_{\mathrm{ad}}=\sum_{i}-\delta_{i} U_{\mathrm{b}} \theta\left(l_{\mathrm{b}} / 2-\left|l_{i}-l_{\mathrm{c}}\right|\right),
$$

where the square-well binding potential has the depth $U_{\mathrm{b}}$ and the width $l_{\mathrm{b}} . l_{\mathrm{c}}$ is the average length of the receptor-ligand 
complex. $\delta_{i}$ is 1 if the two patches at $i$ are occupied by receptor and ligand molecules, respectively, and otherwise 0 . The Heaviside step function $\theta(x)$ implies that a complex can not be formed if the two protein molecules are separated beyond the binding range. In addition, there are hard-core repulsions to prevent the penetration of the two membranes and to exclude two receptors or ligands from one membrane patch.

A lipid raft domain is described by a cluster of adjacent square patches, as depicted in orange in Fig. 1(b). Each raft domain moves along the membrane surfaces. To take into account the fact that protein molecules prefer raft domains of the membranes, ${ }^{32,38}$ we introduce the energy gain $\Delta U$ for a receptor or ligand to move from the non-raft region of the membranes to a raft domain, i.e. the raft affinity to proteins. Since the raft domains are dynamically floating and do not coalesce over a certain period of time, we impose hard-core repulsions between any two rafts in each membrane. This also allows us to study how the raft size plays a role in biomembrane adhesion.

We employ the standard Metropolis MC method to simulate the membrane systems. There are three types of MC trial moves: (i) vertical displacement of the membrane patches to mimic the thermal shape fluctuations, (ii) lateral translation of protein patches, and (iii) the diffusion of the raft domains. The proportion of those trial moves in each MC step is chosen according to the physical time scales. The relaxation time of membrane shape fluctuations is about 1.0 microsecond on the length scale $a=10 \mathrm{~nm},{ }^{39}$ which is set to match the exclusion radius of membrane proteins. ${ }^{40}$ The time for proteins to diffuse along the membrane surfaces over a distance of $10 \mathrm{~nm}$ is of the order of 10 microseconds as estimated by $t=a^{2} / 4 D_{\mathrm{p}}$ with $D_{\mathrm{p}} \sim 1 \mu \mathrm{m}^{2} \mathrm{~s}^{-1} \cdot{ }^{41-43}$ Since the raft domains enriched in saturated lipids are more viscous than the non-raft regime consisting of unsaturated lipids, the diffusion of the proteins will be slowed down by a factor $\rho=0.01-0.5$ if they move from a non-raft region into a raft. ${ }^{43,44}$ Here, we choose $\rho=0.1$ similar to the ratio of diffusion coefficients of lipid molecules in raft and non-raft domains. ${ }^{45}$ The diffusive coefficient $D_{\mathrm{r}}$ of a raft domain is smaller than that of proteins $D_{\mathrm{p}}$ due to its larger size and can be estimated from the Saffman-Delbrück equation ${ }^{46}$

$$
\frac{D_{\mathrm{r}}}{D_{\mathrm{p}}}=\left(\ln \frac{\mu \ell_{\mathrm{me}}}{\mu^{\prime} R_{\mathrm{r}}}-\gamma\right) /\left(\ln \frac{\mu \ell_{\mathrm{me}}}{\mu^{\prime} R_{\mathrm{p}}}-\gamma\right)
$$

where the viscosity of the membrane $\mu=1$ poise, the viscosity of the surrounding fluid $\mu^{\prime}=0.01$ poise, the Euler's constant $\gamma=0.5772,{ }^{46}$ and the thickness of the membranes $\ell_{\text {me }}=5 \mathrm{~nm}^{43}$ $R_{\mathrm{p}}$ and $R_{\mathrm{r}}$ are the radii of the protein molecules and raft domains, respectively. The exact ratio of diffusion coefficients for proteins and raft domains does not affect the binding constant $K$, since $K$ is an equilibrium quantity and does not depend on the dynamic properties of the system. Then in one MC step we attempt to move all the lattice sites 10 times, all the proteins in non-raft domains (a)

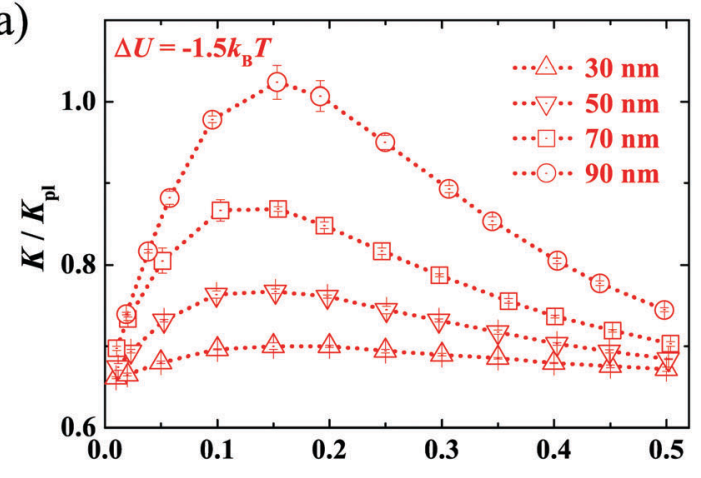

(c)

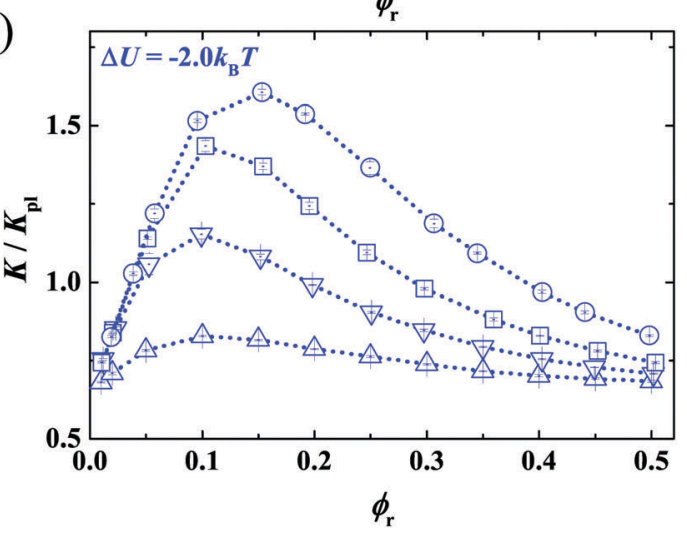

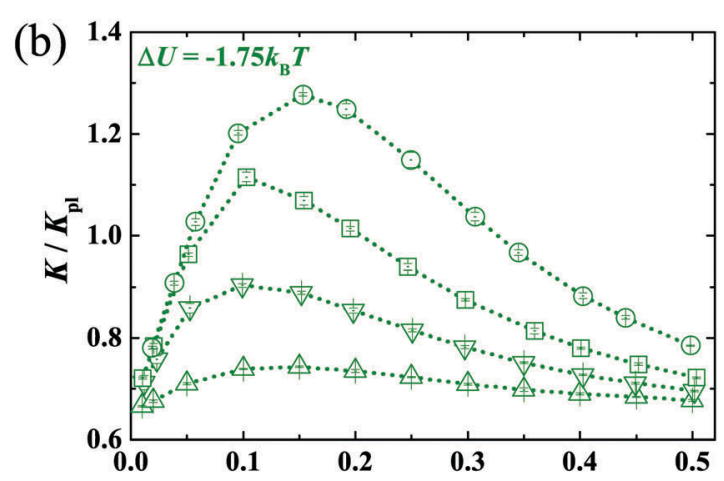

(d)

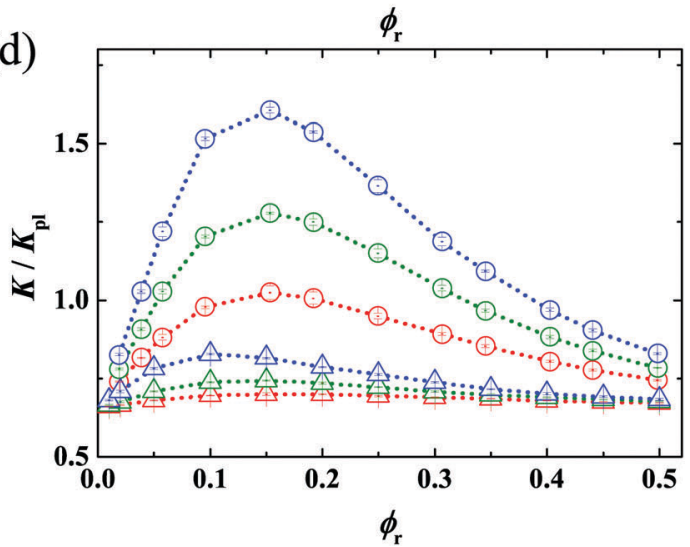

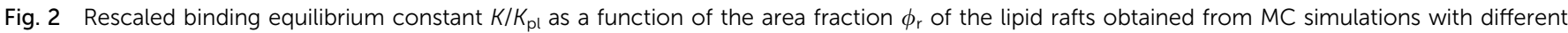

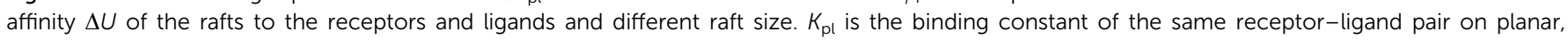
supported membranes without shape fluctuations. The dotted lines are guides for the eye. 
once, each protein in raft domains with a probability of 0.1 , and each raft domain with a probability determined by eqn (4). The attempt frequencies are set to match the time scales of the underlying physical motions. ${ }^{47}$ The trial moves will be accepted or rejected based on the Metropolis algorithm. A receptor-ligand complex is identified if a receptor and a ligand occupy opposing lattices of the two membranes and interact within the binding potential (eqn (3)). The binding constant $K$ is then calculated from the area concentrations according to eqn (1).

We simulate two tensionless membranes each with an area of $600 \times 600 \mathrm{~nm}^{2}$ in a cubic box under periodic boundary conditions. The membrane size is much larger than the parallel correlation length (see Fig. 12), so that the finite size effect is negligible. For the square-well binding potential, the binding energy $U_{\mathrm{b}}=5 k_{\mathrm{B}} T$, the binding range $l_{\mathrm{b}}=1 \mathrm{~nm}$, and the average complex length $l_{\mathrm{c}}=15 \mathrm{~nm} .^{20,48}$ The bending rigidity of lipid membranes $\kappa$ is typically $10 k_{\mathrm{B}} T{ }^{22}$ We choose $\kappa=10 k_{\mathrm{B}} T$ for the non-raft membrane patches, and 10 or $20 k_{\mathrm{B}} T$ for the raft domains. ${ }^{49}$ The typical protein concentration in the cell membrane is several hundred molecules per square micrometer. ${ }^{20}$ In our simulations the protein number in each membrane $N_{\mathrm{p}}=200$ unless otherwise specified. The size of lipid raft domains has been estimated experimentally to be around $30-100 \mathrm{~nm} .^{50}$ The protein concentration in lipid rafts is around $10^{3} \sim 5 \times$ $10^{3}$ molecules $\mu \mathrm{m}^{-2} .^{38}$ To obtain this typical protein concentration in raft domains, the raft affinity $\Delta U$ is set to be in the range from -2.0 to $-1.5 k_{\mathrm{B}} T$. Since the intercellular interaction process is accompanied by the variation of raft concentration in the membrane contact zone, ${ }^{32}$ we vary the area fraction of the raft domains up to $50 \%$ of the membrane surface area according to ref. 31 and 51 .

\section{Results and discussion}

The preferential partitioning of receptor and ligand proteins on the raft domains affects the receptor-ligand binding. As shown in Fig. 2(a)-(c), the binding constant $K$ varies with the area fraction $\phi_{\mathrm{r}}$ of the rafts, and there exists a maximal $K$ at a certain value of $\phi_{\mathrm{r}}$ between about 0.1 and 0.2 . Surprisingly, this maximum can even be greater than the binding constant $K_{\mathrm{pl}}$ of the same receptor and ligand molecules on planar, supported membranes without thermally-excited shape fluctuations; see Fig. 7 for the computation of $K_{\mathrm{pl}}$. This is contrary to the case of homogeneous membranes, where $K$ is weakened by the thermal fluctuations of the membranes and therefore is less than $K_{\mathrm{pl}} \cdot{ }^{20,21,23}$ Fig. $2(\mathrm{~d})$ compares the binding constant at different affinities of the rafts to proteins $\Delta U=-1.5$ (red), -1.75 (green), -2.0 (blue) $k_{\mathrm{B}} T$ for the domain size of 30 (triangle) and 90 (circle) nm. Larger raft domains and stronger (a)

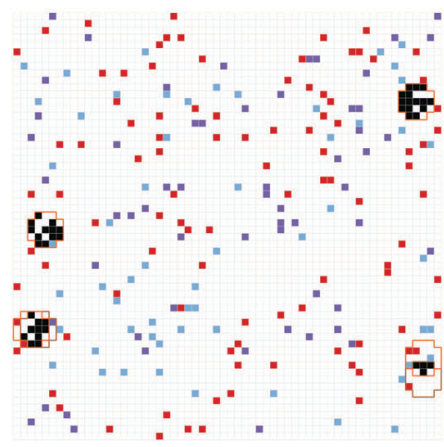

$d=50 \mathrm{~nm} \phi_{\mathrm{r}}=0.02$

(d)

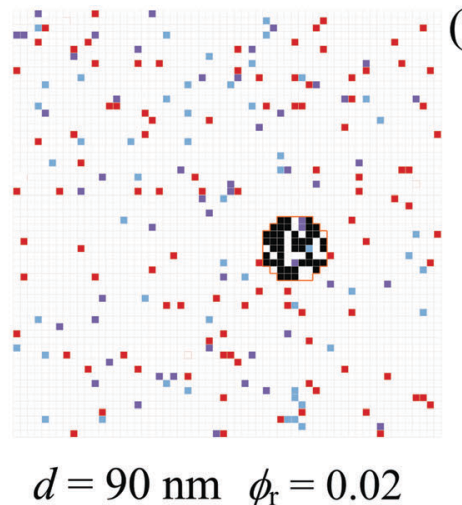

(b)

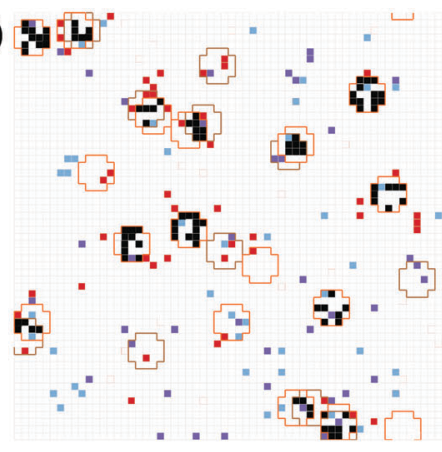

$d=50 \mathrm{~nm} \quad \phi_{\mathrm{r}}=0.1$ (c)

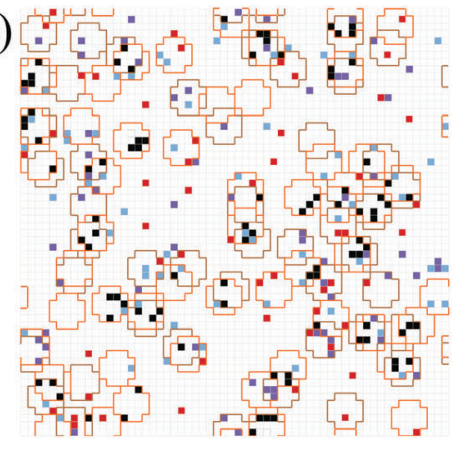

$d=50 \mathrm{~nm} \phi_{\mathrm{r}}=0.3$ (e)

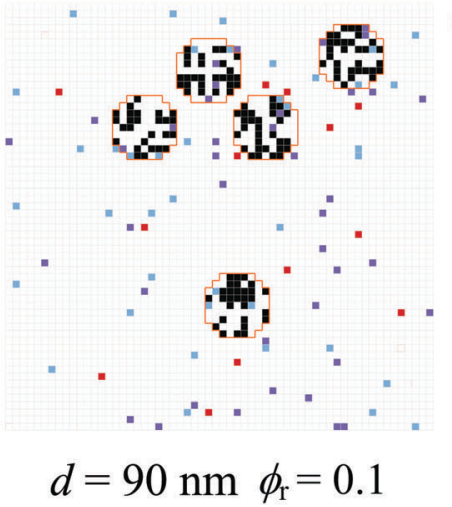

(f)

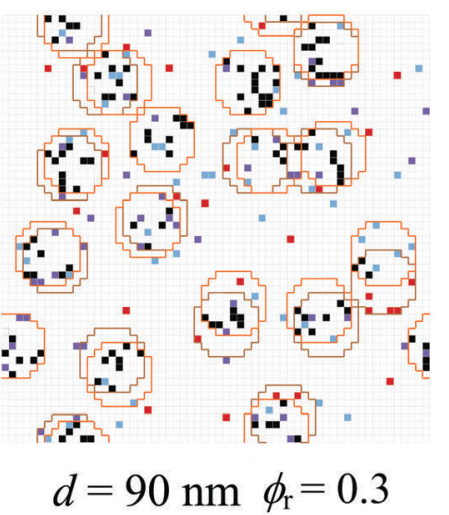

Fig. 3 Top view snapshots of the simulated membranes with different diameters $d$ and area fraction $\phi_{\mathrm{r}}$ of the lipid rafts. The dark and light orange lines enclose the raft domains on the two membranes, respectively. The light purple and blue membrane patches are with free receptors and ligands, the black with bound receptors and ligands both in raft domains, and the red with the rest of the receptor-ligand complexes. 
raft affinity lead to greater $K$. Such enhancement can be readily detected in experiments of multicomponent membrane systems consisting of saturated and unsaturated phospholipids as well as cholesterol.

The dependence of the binding constant $K$ on the area fraction of the lipid rafts can be understood from the thermal fluctuations of the membranes. Fig. 3 shows the snapshots of the simulated membranes with different area fractions and sizes of the rafts viewed from the top. The raft domains on the two apposing membranes are those square patches enclosed by the dark and light orange lines, respectively. The light purple and blue patches are free receptors and ligands, the black patches are bound receptors and ligands both in raft domains, and the red patches are the rest of the formed receptor-ligand complexes. The receptor-ligand complexes act as constraints on the local separation of the two membranes since a receptor binds to a ligand only when they are opposing each other and interacting within the range of the binding potential. Adjacent complexes might be regarded as one effective constraint. When there are too few or too many raft domains, the shape fluctuations of the membranes will be significantly suppressed because of the large amount of effective constraints within the non-raft or raft region, as seen from Fig. 3(a) and (c), or (d) and (f). There appears an optimal value of raft area fraction, at which the complexes are mainly accommodated by the rafts and the amount of effective constraints would be minimal. Thereby, the fluctuations of the membranes enhance the receptor-ligand binding, leading to a maximal $K$ possibly greater than in the case of planar membranes without shape fluctuations. A comparison of the upper and lower snapshots in Fig. 3 suggests that, for the same area fraction, larger rafts accommodate more complexes, leading to less effective constraints and larger binding constants. In the simulations, we considered approximately circular, square and rectangular shapes of the raft domains, which give nearly the same results, see Fig. 8.

To quantitatively see how the receptor-ligand binding is enhanced by the thermal fluctuations of the membranes, we plot the rescaled binding constant $K / K_{\mathrm{pl}}$ as a function of the relative roughness $\xi_{\perp}$ of the two membranes in Fig. 4. Here, the relative roughness $\xi_{\perp}=\sqrt{\left\langle\left(l_{i}-\bar{l}\right)^{2}\right\rangle}$ is defined as the standard deviation of the separations $l_{i}$ at each two apposing square patches from the average value $\bar{l}=\left\langle l_{i}\right\rangle,{ }^{21}$ and thus measures how strong the fluctuations of the two membranes are. The larger the roughness, the stronger the fluctuations and the more configurations the membranes can adopt. In our simulations of two $600 \mathrm{~nm} \times 600 \mathrm{~nm}$ tensionless membranes each with 200 proteins, the roughness is controlled by the area fraction and size of the rafts as well as the affinity of the rafts to the receptors and ligands. Fig. 4 clearly shows that the binding constant increases with the membrane roughness. Surprisingly, at a rather small roughness of half one nanometer, $K$ can exceed the binding constant $K_{\mathrm{pl}}$ for the same receptors and ligands anchored to the planar, supported membrane without shape fluctuations. For receptors and ligands in homogeneous membranes, $K_{\mathrm{pl}}$ is the maximal binding constant and $K$ decreases with increasing membrane roughness since the formation of complexes constrains

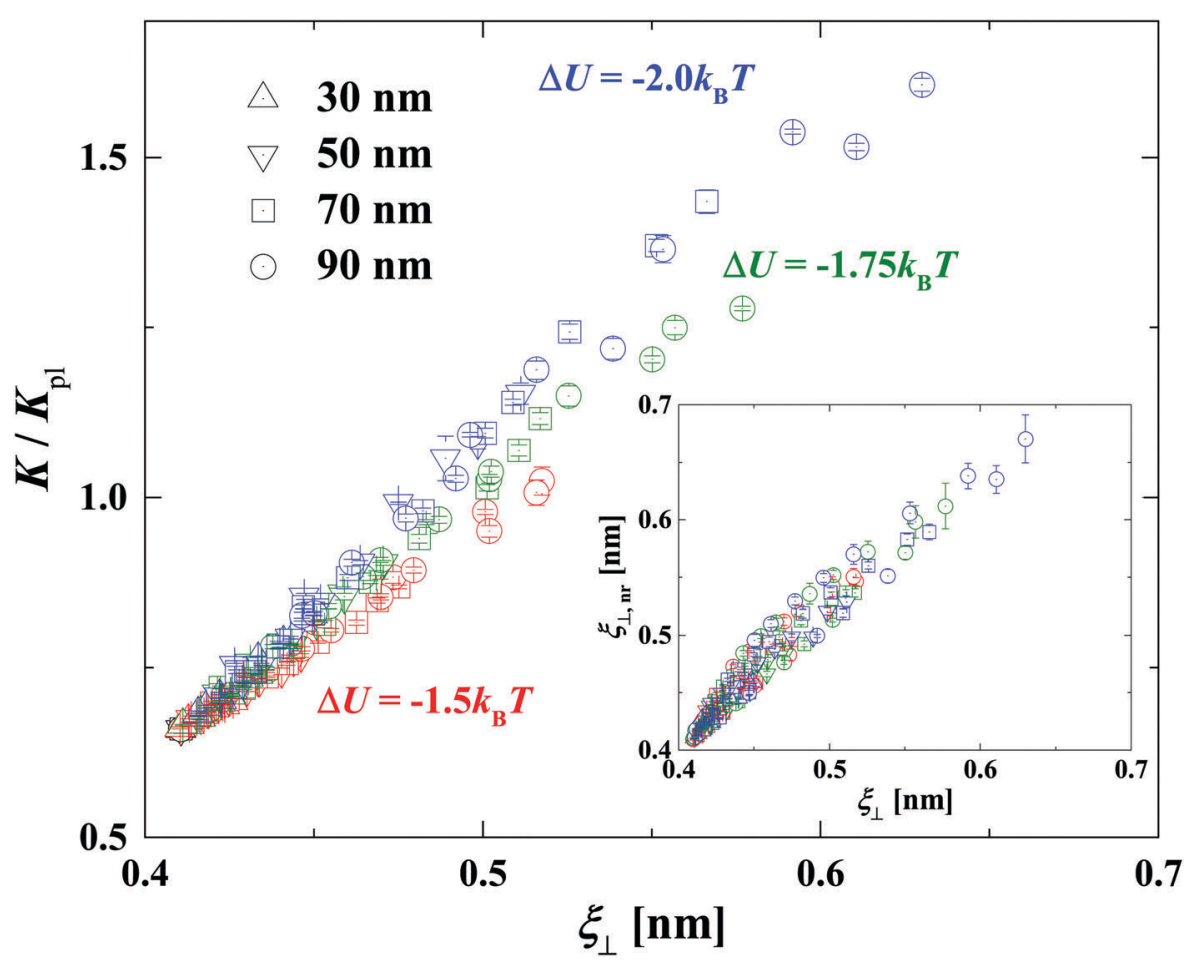

Fig. 4 Rescaled receptor-ligand binding constant $K / K_{\mathrm{pl}}$ as a function of the relative roughness $\xi_{\perp}$ of the two membranes obtained from MC simulations with different area fractions and sizes of the lipid rafts and different raft affinities to proteins for a fixed number $N_{p}=200$ of receptors or ligands anchored to each membrane. Inserted is the relative roughness $\xi_{\perp \text {,nr }}$ of the non-raft region plotted against the roughness $\xi_{\perp}$ of the whole membranes. 
the membrane fluctuations. Then a natural question arises why the fluctuations help in enhancing the binding in the presence of lipid rafts. The inserted plot in Fig. 4 indicates that the relative roughness $\xi_{\perp \text {,nr }}$ of the non-raft regions in the two membranes is very close to the roughness $\xi_{\perp}$ of the whole membranes, suggesting that the fluctuations of the two adhering membranes mainly come from their non-raft regions. Because the proteins prefer the raft domains in the membranes, the overall membranes can take more configurations by having more complexes within or near the rafts. This argument is in agreement with the result shown in Fig. 4 that a stronger raft affinity to the proteins yields a somewhat larger $K$ even for the same roughness.

We've also measured the binding constant for different initial concentrations of the receptors and ligands. As shown in Fig. 5(a), the binding constant increases with the relative roughness of the membranes for each protein number $N_{\mathrm{p}}$. The lowest data points at each $N_{\mathrm{p}}$ are obtained for the raft area fraction $\phi_{\mathrm{r}}=0.01$, and can be well fitted to the solid line given by $K / K_{\mathrm{pl}}=\left[1+\left(\xi_{\perp} / \xi_{0}\right)^{2}\right]^{-1 / 2}$ with the length scale $\xi_{0}=0.35 \mathrm{~nm}$. This relation between $K$ and $\xi_{\perp}$ holds for the receptor-ligand binding in homogeneous membranes without rafts, ${ }^{21,23}$ and is still valid here since the effect from protein partitioning in the small amount of rafts is negligible. Fig. 5(b) shows that $K$ increases with $N_{\mathrm{p}}$ given the same raft area fraction and size, indicating cooperative binding in the presence of rafts. This cooperativity follows the same reasoning as that in the homogeneous membrane case: the membrane shape fluctuations are suppressed with increasing protein concentration (see Fig. 9 for the membrane roughness $\xi_{\perp}$ at different numbers of anchored proteins), facilitating the formation of additional complexes. We obtained similar results for $\Delta U=-1.5$ and $-1.75 k_{\mathrm{B}} T$, as shown in Fig. 10 and 11.

The lipid rafts often exhibit larger bending rigidity than the non-raft regions. ${ }^{49}$ This bending rigidity contrast affects the binding constant of the anchored receptors and ligands. Fig. 6(a) shows that the binding constant for $\kappa_{\mathrm{r}}=2.0 \kappa_{\mathrm{nr}}=$ $20 k_{\mathrm{B}} T$ (points) is slightly larger than that for $\kappa_{\mathrm{r}}=\kappa_{\mathrm{nr}}=10 k_{\mathrm{B}} T$
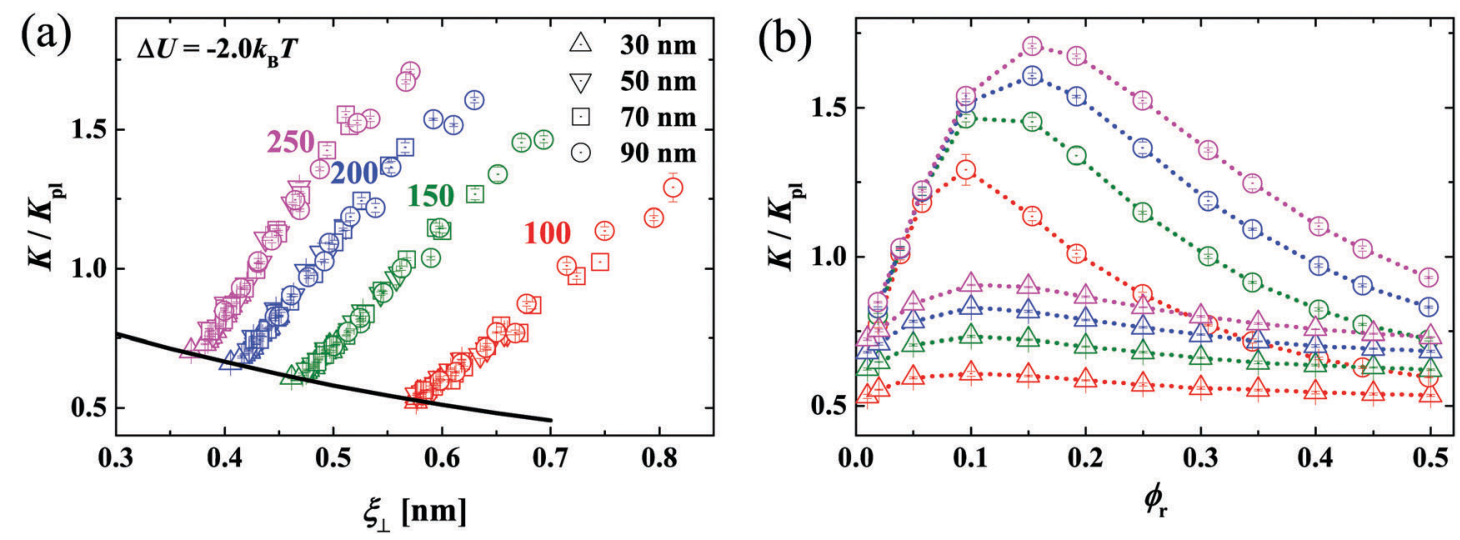

Fig. 5 (a) Rescaled receptor-ligand binding constant $K / K_{\mathrm{pl}}$ as a function of the relative roughness $\xi_{\perp}$ of the two adhering membranes each carrying $N_{\mathrm{p}}=$ $100,150,200$, or 250 proteins for the affinity $\Delta U=-2.0 k_{\mathrm{B}} T$ of the raft domains to the proteins. The results shown in Fig. $2-4$ are from the membrane system with $N_{\mathrm{p}}=200$. The solid line is a least-square fit of the lowest data points for each $N_{\mathrm{p}}$ to the functional form $K / K_{\mathrm{pl}}=\left[1+\left(\xi_{\perp} / \xi_{\mathrm{o}}\right)^{2}\right]^{-1 / 2}$ with the length scale $\xi_{0}=0.35 \mathrm{~nm}$. (b) Rescaled receptor-ligand binding constant $K / K_{\mathrm{pl}}$ as a function of the area fraction $\phi_{\mathrm{r}}$ of the lipid rafts with size of 30 and $90 \mathrm{~nm}$ for different numbers of anchored proteins $N_{p}=100,150,200$, and 250 .
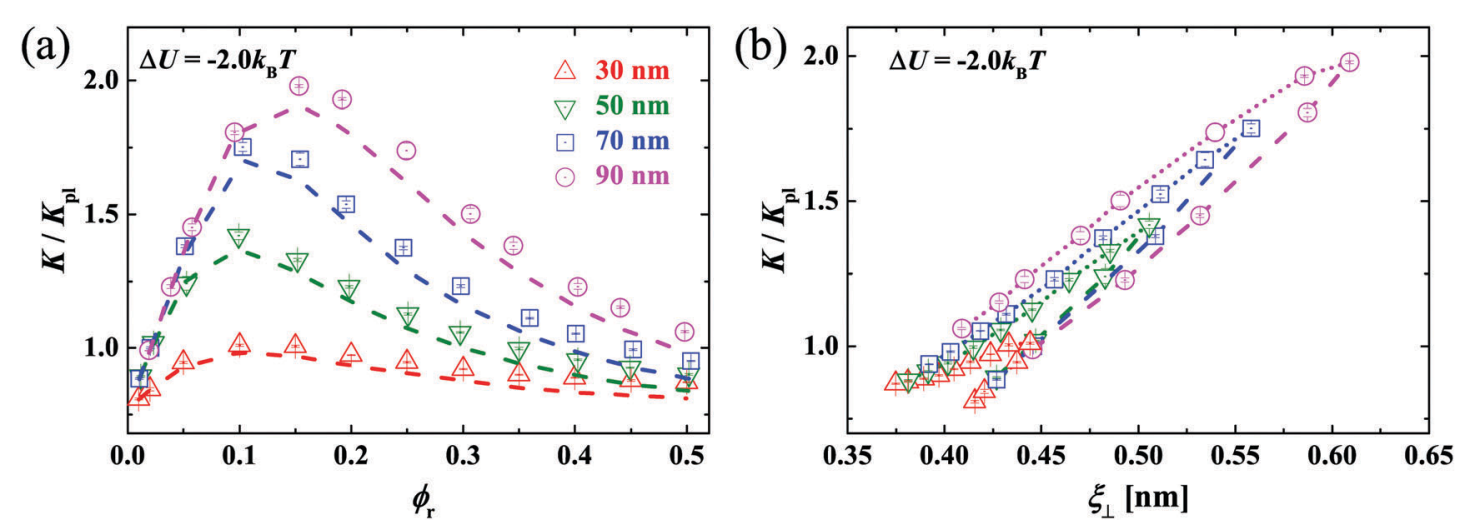

Fig. 6 (a) Rescaled binding constant $K / K_{\mathrm{pl}}$ as a function of the area fraction $\phi_{\mathrm{r}}$ of the raft domains with bending rigidity two times that of the non-raft regions, i.e. $\kappa_{\mathrm{r}}=2.0 \kappa_{\mathrm{nr}}$ and the affinity of rafts to proteins $\Delta U=-2.0 k_{\mathrm{B}} T$. For comparison, the dashed lines are from systems with the same parameters except for $\kappa_{\mathrm{r}}=\kappa_{\mathrm{nr}}$. (b) The binding constant $K / K_{\mathrm{pl}}$ against the relative roughness $\xi_{\perp}$ of the membranes. As the raft area fraction $\phi_{\mathrm{r}}$ increases, $K$ increases with the roughness $\xi_{\perp}$ up to the maximum, following the dashed lines. With further increasing $\phi_{\mathrm{r}}, K$ and $\xi_{\perp}$ decrease as indicated by the dotted lines. 
(dashed lines) at the same raft area fraction $\phi_{\mathrm{r}}$. The contribution of raft domains to the increase of binding constant is twofold here. The thermal shape fluctuations of the rigid raft domains are suppressed, facilitating the formation of receptor-ligand complexes in the domains. The rafts tend to aggregate in order for the more flexible non-raft regions to access more configurations. The binding constant is plotted against the relative roughness $\xi_{\perp}$ of the two membranes in Fig. $6(b) . K$ increases up to the maximum with the roughness as the raft area fraction $\phi_{\mathrm{r}}$ increases, shown by the dashed lines. As $\phi_{\mathrm{r}}$ further increases, $\xi_{\perp}$ and $K$ decrease, following the dotted lines. Different from the result in Fig. 4 with the same bending rigidity for the raft and non-raft domains, the roughness is not the only parameter controlling the binding constant in the general case with the bending rigidity difference, since the aggregation of the more rigid raft domains should be taken into account in a theoretical

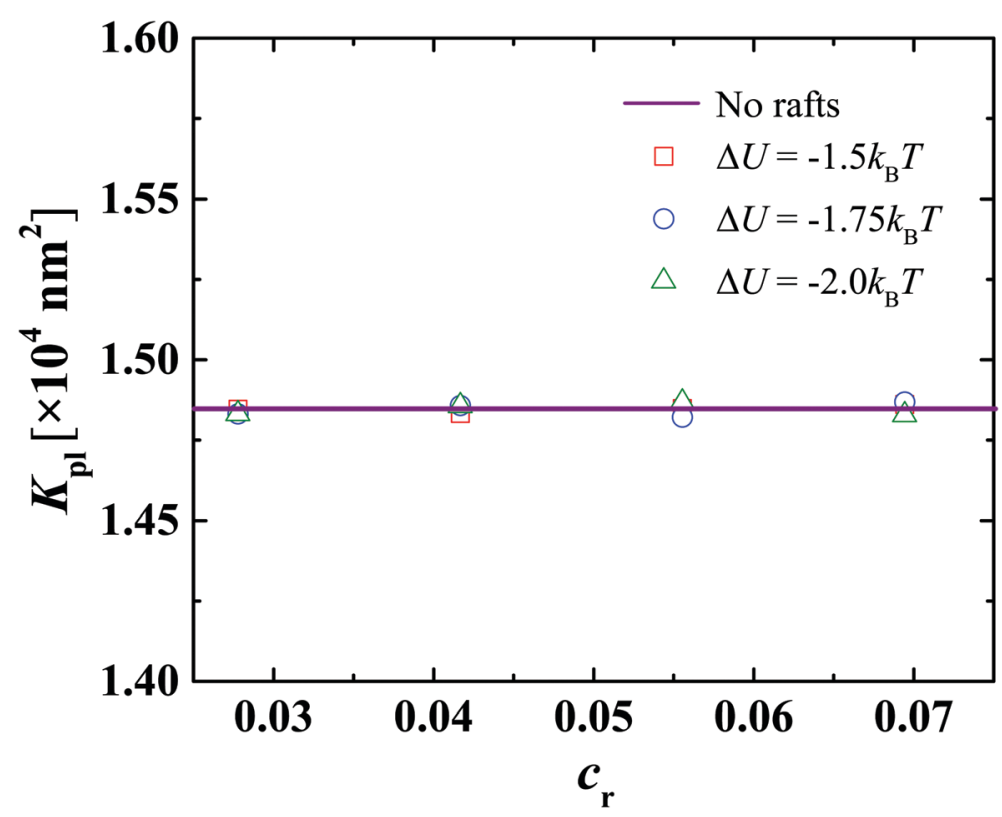

Fig. 7 Binding constant $K_{\mathrm{pl}}$ as a function of protein concentration in rafts $c_{r}$ for different raft affinities for planar, supported membranes without shape fluctuations. Here, lipid rafts with a diameter of $50 \mathrm{~nm}$ make up $25 \%$ of the membrane.

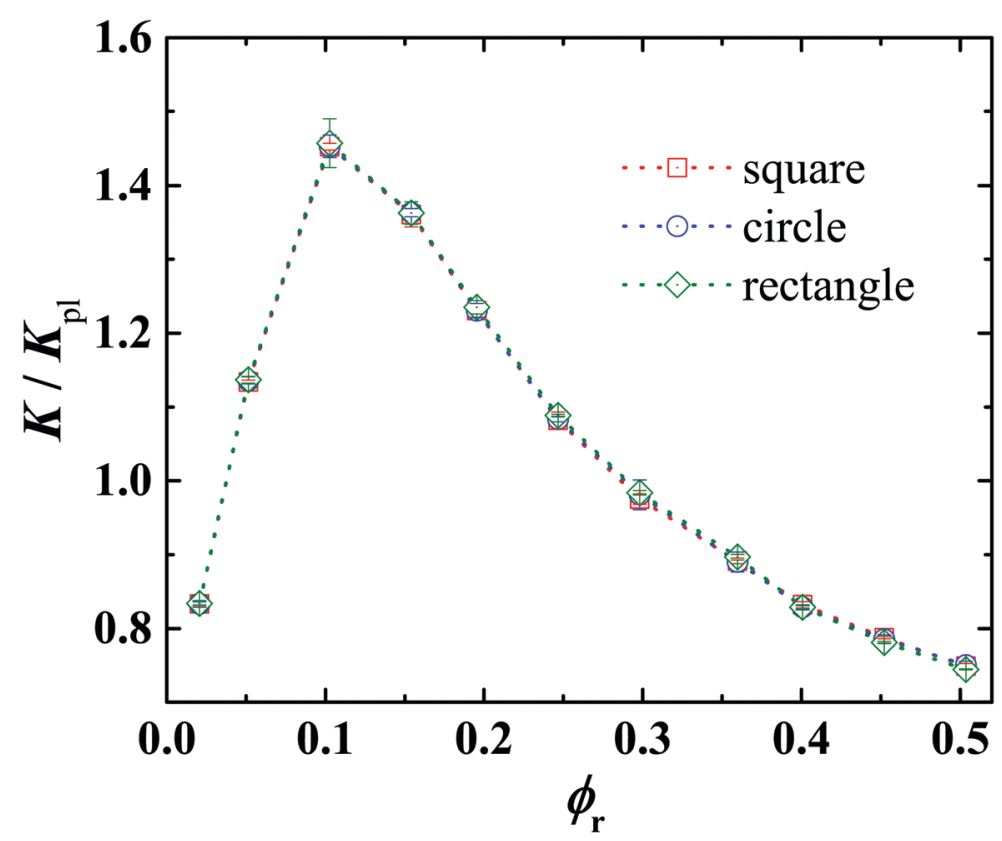

Fig. 8 Rescaled binding constant $K / K_{\mathrm{pl}}$ as a function of the area fraction $\phi_{\mathrm{r}}$ of the raft domains in different shapes with the same area of $3600 \mathrm{~nm}^{2}$. The width-to-length ratio for a rectangular raft is $4: 9$. The affinity of raft domains to the proteins is $\Delta U=-2.0 k_{\mathrm{B}} T$. 
consideration. We note that the relative roughness $\xi_{\perp}$ is a characteristic length in the direction perpendicular to the average plane of the membranes, and the parallel correlation length $\xi_{\|}$ in the direction parallel to the average plane. As shown in Fig. 12, $\xi_{\|}$is proportional to $\xi_{\perp}$, as in the case of homogeneous membranes, suggesting that $\xi_{\|}$and $\xi_{\perp}$ might not be regarded as two independent parameters in theoretical description of the binding constant of receptors and ligands anchored to multicomponent membranes.

\section{Conclusions and outlook}

We have systematically investigated the role of lipid rafts in the binding of membrane-anchored receptors and ligands by using Monte Carlo simulations of a mesoscopic model. We find that the binding constant of membrane-anchored receptor and ligand molecules is enhanced by the presence of lipid rafts, consistent with the experimental observation ${ }^{4,34,35}$ that lipid rafts facilitate TCR-pMHC binding. This enhancement results
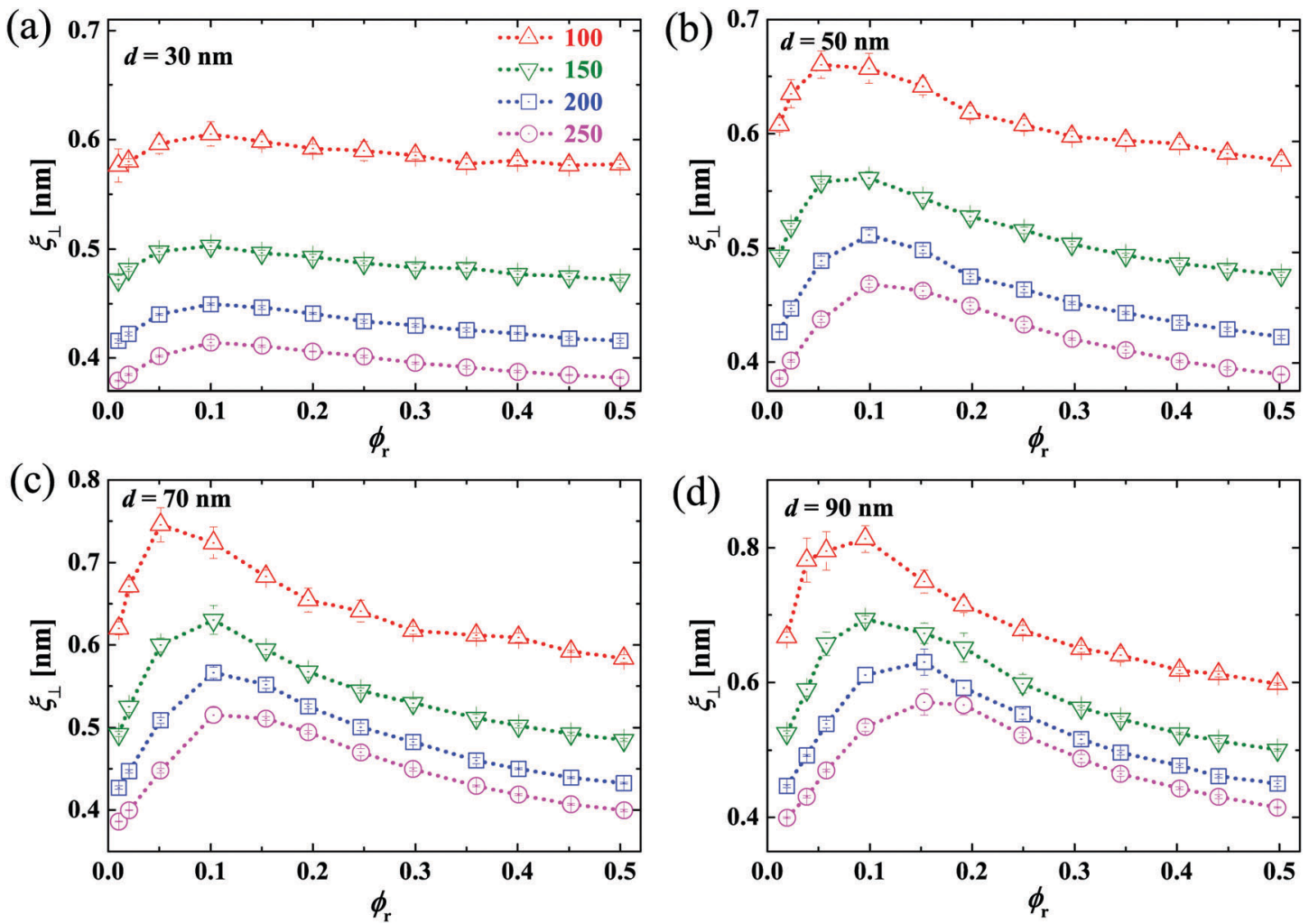

Fig. 9 The relative roughness $\xi_{\perp}$ of the two membranes each carrying $N_{\mathrm{p}}=100,150,200$, or 250 proteins as a function of the area fraction $\phi_{\mathrm{r}}$ of the lipid rafts with different diameters $d$ for raft affinity $\Delta U=-2.0 k_{\mathrm{B}} T$.
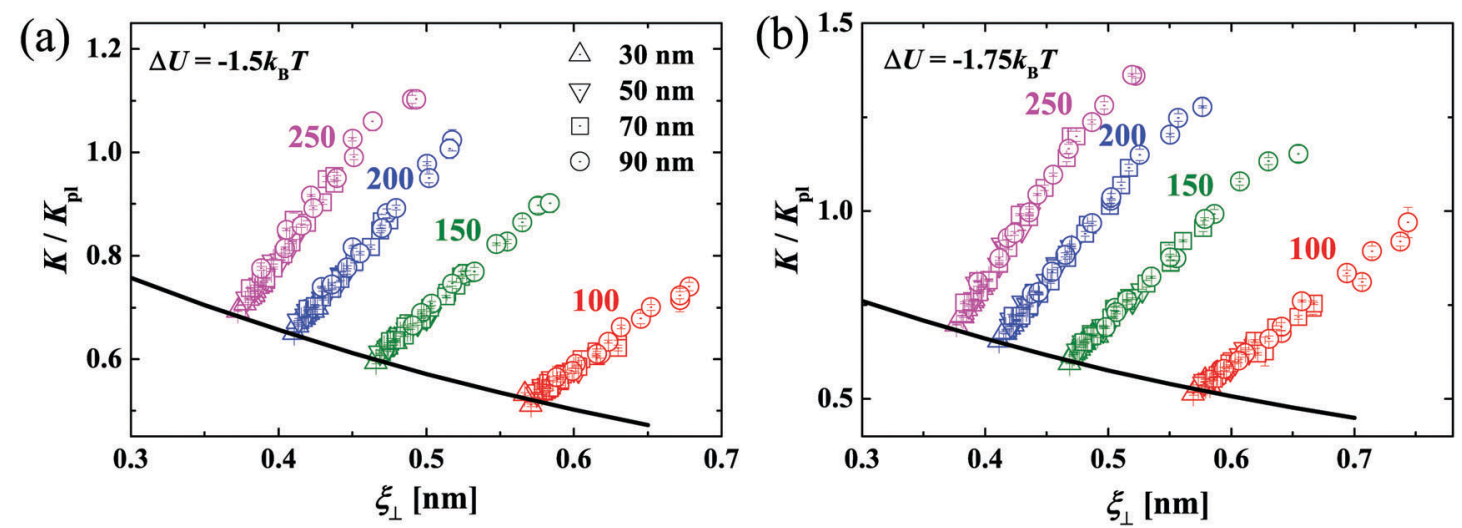

Fig. 10 Rescaled receptor-ligand binding constant $K / K_{\mathrm{pl}}$ as a function of the relative roughness $\xi_{\perp}$ for raft affinity $\Delta U=-1.5$ and $-1.75 k_{\mathrm{B}} T$ and different protein numbers ranging from 100 to 250 in each membrane. The solid lines are least-square fits of the lowest data points for each $N_{p}$ to the functional form $K / K_{\mathrm{pl}}=\left[1+\left(\xi_{\perp} / \xi_{0}\right)^{2}\right]^{-1 / 2}$ with the length scale $\xi_{0}=0.35 \mathrm{~nm}$. 
from the thermal shape fluctuations of non-raft regions of the membranes by having receptor-ligand complexes largely accommodated in the raft domains. Contrary to the case of homogeneous membranes where the binding of receptor and ligand molecules is weakened by the fluctuations of the membranes, the membrane roughness actually functions as a positive regulator for the binding in collaboration with lipid rafts. In the experimental range of parameters we surprisingly find that the binding constant can even be substantially larger than that of the same receptors and ligands for two planar, supported membranes without thermal shape fluctuations. For the lipid raft domains often more rigid than the non-raft regions of the membranes, their clustering further contributes to the aggregation of the anchored receptor and ligand proteins,
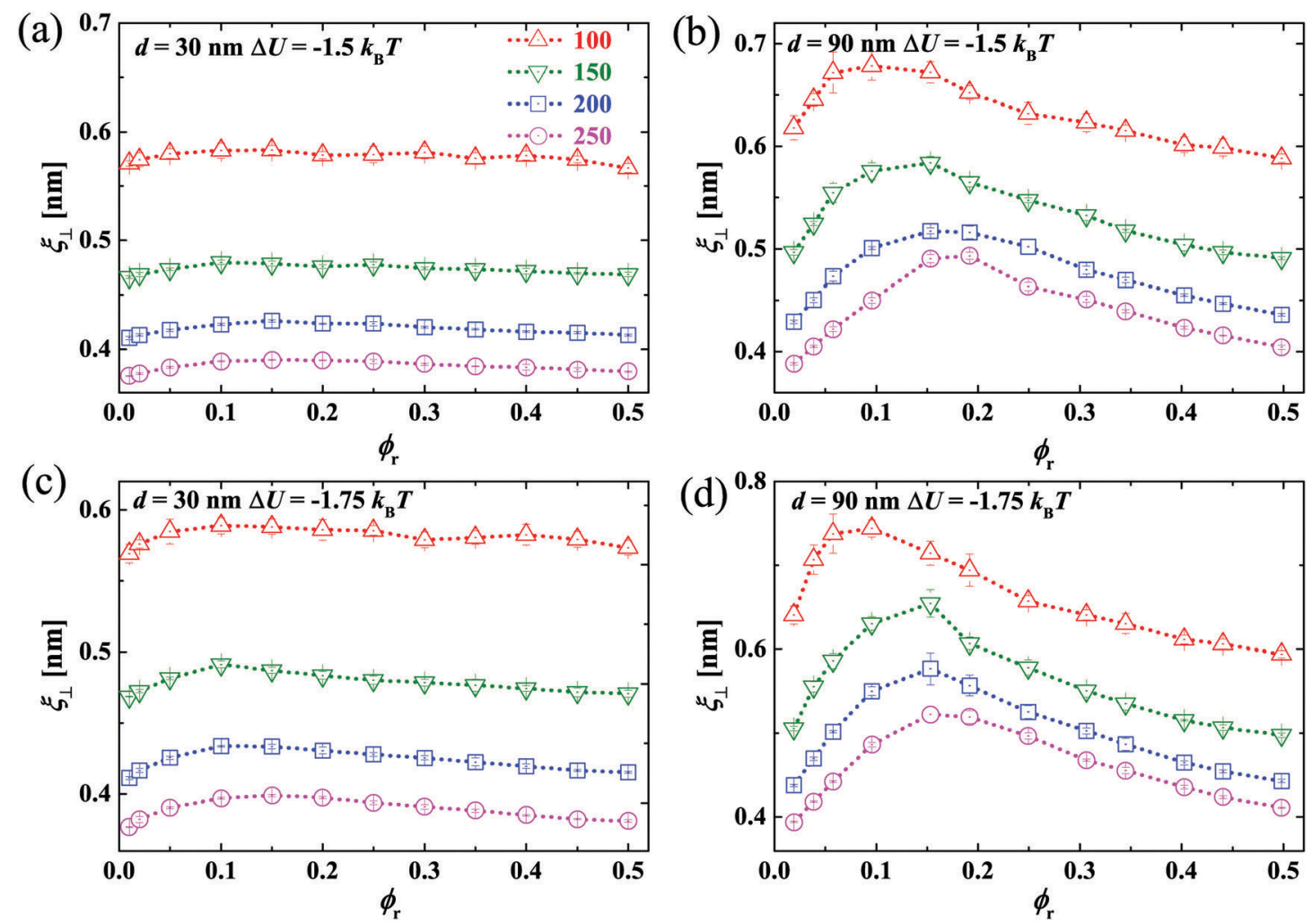

Fig. 11 The relative roughness $\xi_{\perp}$ of the two membranes each carrying $N_{\mathrm{p}}=100,150,200$, or 250 proteins as a function of the area fraction $\phi_{\mathrm{r}}$ of the lipid rafts with diameters of 30 and $90 \mathrm{~nm}$ for raft affinity $\Delta U=-1.5$ and $-1.75 k_{\mathrm{B}} T$.
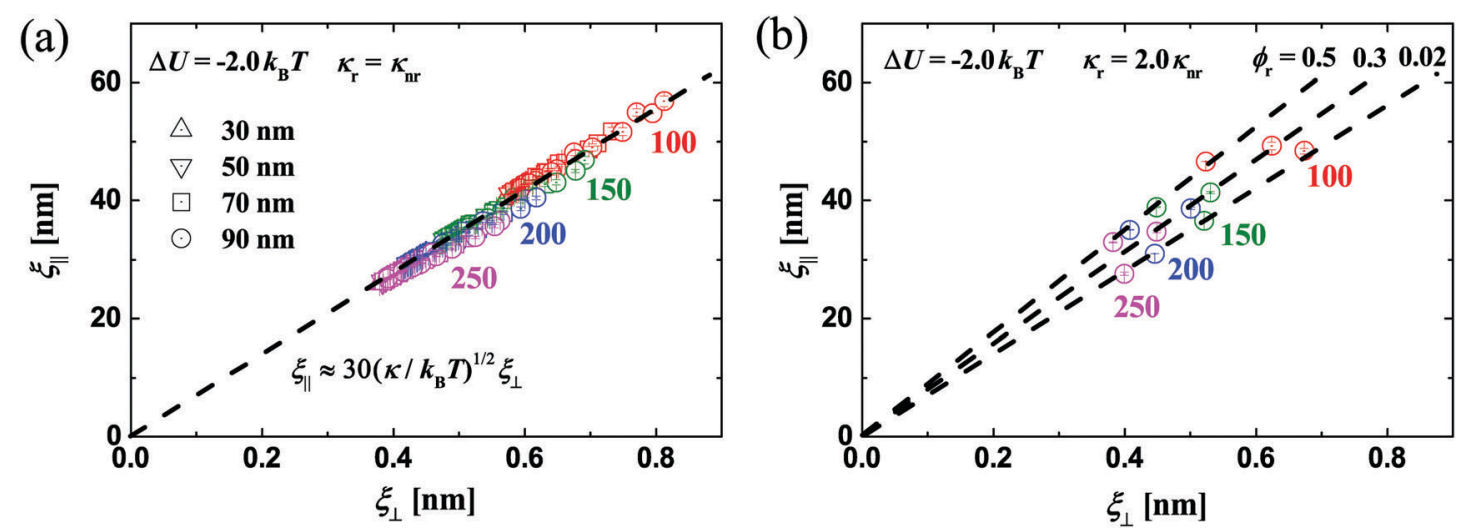

Fig. 12 The parallel correlation length $\xi_{\|}$of the membranes as a function of the relative membrane roughness $\xi_{\perp}$. The parallel correlation length is estimated from $\xi_{\|}=a \exp \left[2 \pi\left(\kappa / k_{\mathrm{B}} T\right)\left\langle(\nabla l)^{2}\right\rangle\right] .^{52}$ (a) The data points are from systems with different raft sizes $(30,50,70$, and $90 \mathrm{~nm})$ and different numbers of membrane-anchored receptors and ligands $(100,150,200$, and 250$)$. The dashed line is the least-square fit $\xi_{\|} \approx 30\left(\kappa / k_{\mathrm{B}} T\right) \xi_{\perp}$ to all the data points, where the bending rigidity $\kappa_{\mathrm{r}}=\kappa_{\mathrm{nr}}$. (b) Each line is the least-square fit to the data points with the same area fraction of lipid rafts $(0.02,0.3$, and 0.5$)$ but different numbers of anchored proteins $(100,150,200$, and 250). The raft has a bending rigidity two times that of the non-raft region. The slope of the fitted line increases with the area fraction of the more rigid raft domains. 
leading to increased binding constant. Our results suggest that cells might regulate the binding of membrane-anchored receptors and ligands by modulating raft characteristics under physiological conditions. We are working on a quantitative description for the dependence of the binding constant on the raft-induced aggregation of the membrane-anchored receptors and ligands. It would also be interesting to see how the lipid rafts affect the binding kinetics of the membrane-anchored proteins.

\section{Acknowledgements}

L. L. thanks T. R. Weikl and Z. K. Lin for insightful discussions. This work was supported by the Programs in the National Natural Science Foundation of China (Grants No. 11472285, 11232013 and 21504038), the Strategic Priority Research Program of the Chinese Academy of Sciences (Grant No. XDB22040102) and the National Key Research and Development Program of China (Grant No. 2016YFA0501601). J. L. Hu also acknowledges the financial support by Fundamental Research Funds for the Central Universities and Shenzhen Science and Technology Innovation Committee (Grant No. JCYJ20160531151105346).

\section{Notes and references}

1 E. Sackmann and A.-S. Smith, Soft Matter, 2014, 10, 1644-1659.

2 P. A. van der Merwe and O. Dushek, Nat. Rev. Immunol., 2011, 11, 47-55.

3 J. B. Huppa, M. Axmann, M. A. Mörtelmaier, B. F. Lillemeier, E. W. Newell, M. Brameshuber, L. O. Klein, G. J. Schütz and M. M. Davis, Nature, 2010, 463, 963-967.

4 J. Huang, V. I. Zarnitsyna, B. Liu, L. J. Edwards, N. Jiang, B. D. Evavold and C. Zhu, Nature, 2010, 464, 932-936.

5 M. L. Dustin, L. M. Ferguson, P. Y. Chan, T. A. Springer and D. E. Golan, J. Cell Biol., 1996, 132, 465-474.

6 D.-M. Zhu, M. L. Dustin, C. W. Cairo and D. E. Golan, Biophys. J., 2007, 92, 1022-1034.

7 T. P. Tolentino, J. Wu, V. I. Zarnitsyna, Y. Fang, M. L. Dustin and C. Zhu, Biophys. J., 2008, 95, 920-930.

8 G. P. O'Donoghue, R. M. Pielak, A. A. Smoligovets, J. J. Lin and J. T. Groves, eLife, 2013, 2, e00778.

9 J. Huang, J. Chen, S. E. Chesla, T. Yago, P. Mehta, R. P. McEver, C. Zhu and M. Long, J. Biol. Chem., 2004, 279, 44915-44923.

10 W. Chen, E. A. Evans, R. P. McEver and C. Zhu, Biophys. J., 2008, 94, 694-701.

11 B. Liu, W. Chen, B. D. Evavold and C. Zhu, Cell, 2014, 157, 357-368.

12 B. T. Marshall, M. Long, J. W. Piper, T. Yago, R. P. McEver and C. Zhu, Nature, 2003, 423, 190-193.

13 B. T. Marshall, K. K. Sarangapani, J. Z. Lou, R. P. McEver and C. Zhu, Biophys. J., 2005, 88, 1458-1466.

14 M. Long, S. Q. Lü and G. Y. Sun, Cell. Mol. Immunol., 2006, 3, 79-86.
15 R. Alon, D. A. Hammer and T. A. Springer, Nature, 1995, 374, 539-542.

16 S. Neelamegham, Cell Commun. Adhes., 2004, 11, 35-50.

17 L. Limozin, P. Bongrand and P. Robert, Sci. Rep., 2016, 6, 35193.

18 M. L. Dustin, S. K. Bromley, M. M. Davis and C. Zhu, Annu. Rev. Cell Dev. Biol., 2001, 17, 133-157.

19 C. Zhu, N. Jiang, J. Huang, V. I. Zarnitsyna and B. D. Evavold, Immunol. Rev., 2013, 251, 49-64.

20 H. Krobath, B. Różycki, R. Lipowsky and T. R. Weikl, Soft Matter, 2009, 5, 3354-3361.

21 J. L. Hu, R. Lipowsky and T. R. Weikl, Proc. Natl. Acad. Sci. U. S. A., 2013, 110, 15283-15288.

22 G. K. Xu, J. L. Hu, R. Lipowsky and T. R. Weikl, J. Chem. Phys., 2015, 143, 243136.

23 J. L. Hu, G. K. Xu, R. Lipowsky and T. R. Weikl, J. Chem. Phys., 2015, 143, 243137.

24 T. R. Weikl, J. L. Hu, G. K. Xu and R. Lipowsky, Cell Adhes. Migr., 2016, 10, 576-589.

25 S. J. Singer and G. L. Nicolson, Science, 1972, 175, 720-731.

26 D. Lingwood and K. Simons, Science, 2010, 327, 46-50.

27 K. Simons and M. J. Gerl, Nat. Rev. Mol. Cell Biol., 2010, 11, 688-699.

28 F. Mollinedo and C. Gajate, Adv. Biol. Regul., 2015, 57, 130-146.

29 B. Alberts, A. Johnson, J. Lewis, M. Raff, K. Roberts and P. Walter, Molecular Biology of the Cell, Garland Science, New York, 2014.

30 C. Zhu, S. L. Das and T. Baumgart, Biophys. J., 2012, 102, 1837-1845.

31 M. Fallahi-Sichani and J. J. Linderman, PLoS One, 2009, 4, e6604.

32 S. K. Pierce, Nat. Rev. Immunol., 2002, 2, 96-105.

33 D. A. Hicks, N. N. Nalivaeva and A. J. Turner, Front. Physiol., 2012, 3, 189.

34 H. A. Anderson, E. M. Hiltbold and P. A. Roche, Nat. Immunol., 2000, 1, 156-162.

35 H. A. Anderson and P. A. Roche, Biochim. Biophys. Acta, 2015, 1853, 775-780.

36 H. Krobath, B. Różycki, R. Lipowsky and T. R. Weikl, PLoS One, 2011, 6, e23284.

37 T. R. Weikl, M. Asfaw, H. Krobath, B. Różycki and R. Lipowsky, Soft Matter, 2009, 5, 3213-3224.

38 K. Simons and D. Toomre, Nat. Rev. Mol. Cell Biol., 2000, 1, 31-39.

39 U. Seifert and S. A. Langer, Biophys. Chem., 1994, 49, 13-22. 40 P. K. Tsourkas, M. L. Longo and S. Raychaudhuri, Biophys. J., 2008, 95, 1118-1125.

41 Y. Gambin, R. Lopez-Esparza, M. Reffay, E. Sierecki, N. S. Gov, M. Genest, R. S. Hodges and W. Urbach, Proc. Natl. Acad. Sci. U. S. A., 2006, 103, 2098-2102.

42 S. Ramadurai, A. Holt, V. Krasnikov, G. van den Bogaart, J. A. Killian and B. Poolman, J. Am. Chem. Soc., 2009, 131, 12650-12656.

43 A. Pralle, P. Keller, E.-L. Florin, K. Simons and J. K. H. Hörber, J. Cell Biol., 2000, 148, 997-1007. 
44 C. Dietrich, B. Yang, T. Fujiwara, A. Kusumi and K. Jacobson, Biophys. J., 2002, 82, 274-284.

45 G. Orädd, V. Shahedi and G. Lindblom, Biochim. Biophys. Acta, 2009, 1788, 1762-1771.

46 P. G. Saffman and M. Delbrück, Proc. Natl. Acad. Sci. U. S. A., 1975, 72, 3111-3113.

47 H. Krobath, G. J. Schütz, R. Lipowsky and T. R. Weikl, Europhys. Lett., 2007, 78, 38003.
48 T. R. Weikl and R. Lipowsky, Phys. Rev. E: Stat., Nonlinear, Soft Matter Phys., 2001, 64, 011903.

49 P. S Niemelä, S. Ollila, M. T Hyvönen, M. Karttunen and I. Vattulainen, PLoS Comput. Biol., 2007, 3, e34.

$50 \mathrm{H}$. Kalthoff, Death receptors and cognate ligands in cancer, Springer, Heidelberg, Dordrecht, New York, 2009.

51 L. J. Pike, J. Lipid Res., 2003, 44, 655-667.

52 R. R. Netz and R. Lipowsky, Phys. Rev. Lett., 1993, 71, 3596-3599. 